\title{
Supergiant Cavernous Carotid Aneurysm: Successful Management with Ligation of Internal Carotid Artery and STA-MCA Bypass
}

\author{
Navneet Singla ${ }^{1}$ Ashish Aggarwal ${ }^{1}$ Kokkula Praneeth ${ }^{1} \quad$ Chirag Ahuja ${ }^{2}$ \\ ${ }^{1}$ Department of Neurosurgery, Post Graduate Institute of Medical \\ Education \& Research (PGIMER), Chandigarh, India \\ 2Department of Radio diagnosis and Imaging, Post Graduate \\ Institute of Medical Education \& Research (PGIMER), Chandigarh, \\ Address for correspondence Ashish Aggarwal, MCh, \\ Department of Neurosurgery, Post Graduate Institute of Medical \\ Education \& Research (PGIMER), Chandigarh 160012, India \\ (e-mail: aaggarwal_7@yahoo.com).
} India

J Neurosci Rural Pract 2019;10:697-699
Abstract
Keywords
- internal carotid artery ligation
- STA-MCA bypass
- super giant ICA aneurysm

Supergiant cerebral aneurysm (size $>6 \mathrm{~cm}$ ) is a rare and challenging cerebrovascular disease with few documented cases in literature. We describe a simple, safe, and effective treatment option for supergiant cavernous carotid aneurysm and discuss the proposed mechanisms for a favorable outcome.

\section{Introduction}

An 18-year-old young male patient having mild intermittent headache for 2 years presented with decreased vision in left eye, severe headache, and features suggestive of increased intracranial pressure for 10 days. On examination, the patient had left nasal field deficit and papilledema. Computed tomography (CT) showed isodense well-circumscribed space occupying lesion (SOL) in left parasellarsuprasellar region with areas of calcifications. Contrast enhanced MR revealed a supergiant aneurysm $(6.1 \times 3.6 \times$ $3.4 \mathrm{~cm}$ ) in cavernous segment of left internal carotid artery (ICA). Digital subtraction angiography (DSA) confirmed the presence of supergiant aneurysm (-Fig. 1A-C) Further, balloon occlusion test (BOT) of left ICA was suggestive of moderate cross flow from right ICA across anterior communicating (Acomm) artery. Patient also had hypothyroidism and hypocortisolism. Multiple treatment options described for giant aneurysm in the literature include direct clipping, excision of aneurysm, gradual ICA ligation, parent vessel occlusion with or without revascularization, trapping of aneurysm with or without revascularization, flow diverter, and coil occlusion of aneurysm..$^{1-5}$ For the present case, the treatment plan was discussed amongst neurosurgeon, endovascular team, and patient. In the presence of raised intracranial pressure and compression on cranial nerves, treatment plan selected was proximal vessel occlusion (PVO) and revascularization i.e., ligation of left ICA in cervical region with superficial temporal artery-middle cerebral artery (STA-MCA) bypass. Postoperative course was uneventful and patient was discharged in the same status. At 3 months follow-up, patient was relieved of headache, papilledema resolved but visual field deficit remained. Follow-up DSA showed occlusion of left ICA, nonfilling aneurysm, and patent left STA-MCA bypass. Angiographic sufficiency of bypass was demonstrated as follows: contrast in left external carotid artery (ECA) run with compression of right common carotid artery, showed filling of right side vessels across Acomm ( - Fig. 1D-F). Supergiant intracranial aneurysms remain a formidable challenge to the neurosurgeons with very few options available. Dogmatic statements cannot be made about treatment strategy and it has to be tailor made for each individual case. The size and the location of this aneurysm precluded direct manipulation and 
clipping. Parent vessel occlusion i.e., ICA ligation removed the pressure head and created a "blind alley formation" for the retrograde flow leading to thrombosis and obliteration of aneurysm. ${ }^{1,2,5}$ STA-MCA bypass augmented the cross flow from right ICA across (Acomm) artery and prevented ischemia in left ICA territory. In cases having even moderate cross flow, ICA ligation with STA-MCA bypass is a good option for supergiant ICA aneurysms.

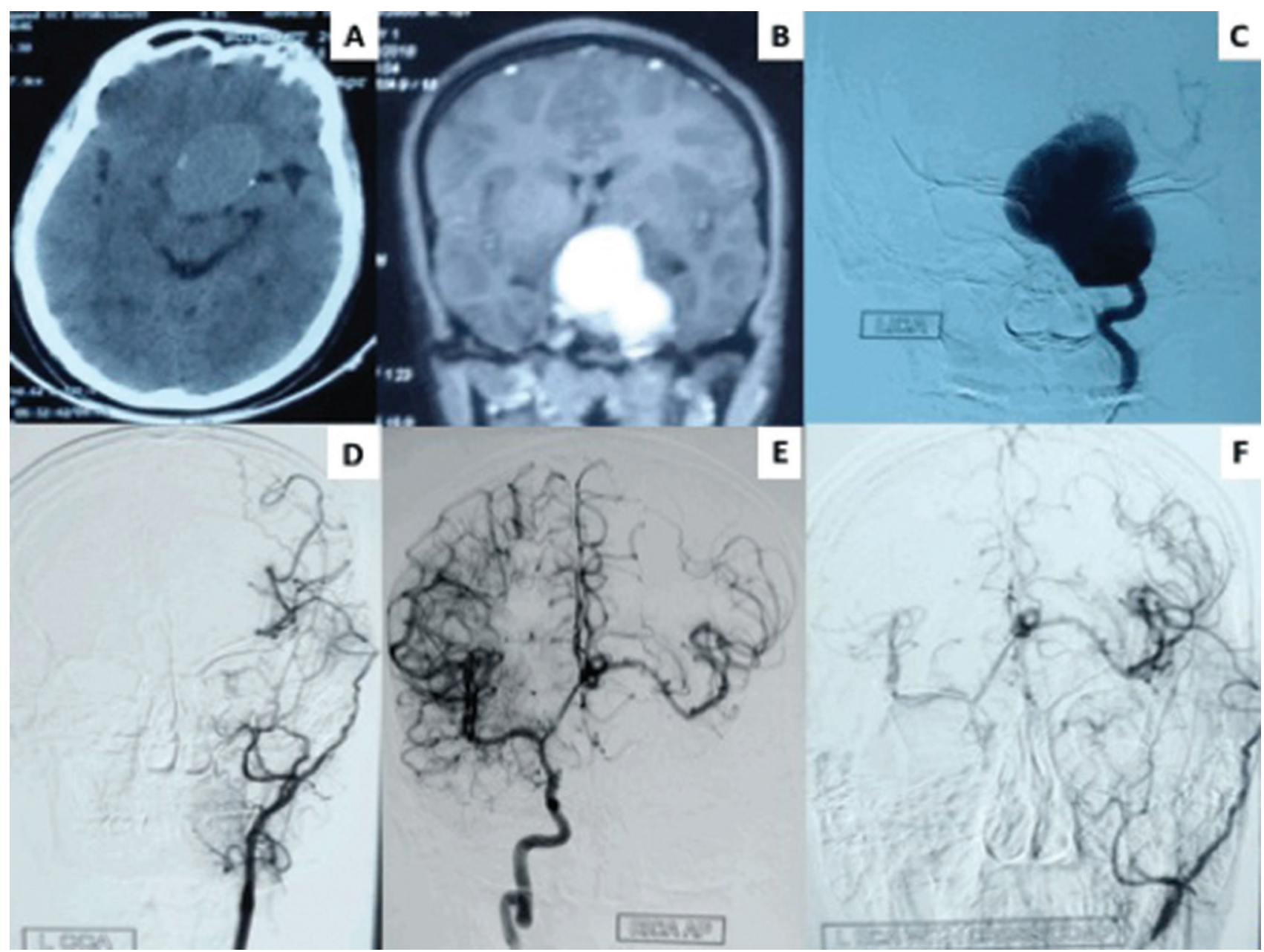

Fig. 1 (A) NCCT head showing isodense well circumscribed space occupying lesion in left parasellar-suprasellar region with areas of calcifications in the wall. (B) Contrast-enhanced MR brain intensely contrast enhancing lesion. (C) Digital subtraction angiography (DSA) showing super giant aneurysm in cavernous segment of left internal carotid artery (ICA). (D) Postoperative DSA run of left common carotid artery showing occlusion of left ICA with patent superficial temporal artery (STA)-middle cerebral artery (MCA) bypass and no retrograde filling of aneurysm. (E) Postoperative DSA run of right ICA showing good cross flow across Anterior communicating (Acomm) artery with no retrograde filling of aneurysm. (F) Postoperative DSA of left external carotid artery with cross compression showing filling of contralateral (right) ACA and MCA from STA-MCA bypass through Acomm artery with no filling of aneurysm. 


\section{Funding}

None.

\section{Conflict of Interest}

None declared.

\section{References}

1 Sriamornrattanakul K, Sakarunchai I, Yamashiro K, et al. Surgical treatment of large and giant cavernous carotid aneurysms. Asian J Neurosurg 2017;12(3):382-388

2 Singla N, Aggarwal A. A giant partially thrombosed, partially filling, and partially calcified intracranial aneurysm. Surg Neurol Int 2018;9:139
3 Jeong YH, Kim JY, Koo YM, et al. Endovascular treatment of giant serpentine aneurysm of the middle cerebral artery. J Cerebrovasc Endovasc Neurosurg 2016;18(3):264-270

4 Nishio A, Sakaguchi M, Murata K, et al. Surgical treatment of supergiant cerebral aneurysms. Surgery for Cerebral Stroke. 1991;19:408-413

5 Amin-Hanjani S, Chen PR, Chang SW, Spetzler RF. Long-term follow-up of giant serpentine MCA aneurysm treated with EC-IC bypass and proximal occlusion. Acta Neurochir (Wien) 2006;148(2):227-228 